\title{
Majority dynamics and aggregation of information in social networks
}

\author{
Elchanan Mossel · Joe Neeman · Omer Tamuz
}

Published online: 11 June 2013

(C) The Author(s) 2013

\begin{abstract}
Consider $n$ individuals who, by popular vote, choose among $q \geq 2$ alternatives, one of which is "better" than the others. Assume that each individual votes independently at random, and that the probability of voting for the better alternative is larger than the probability of voting for any other. It follows from the law of large numbers that a plurality vote among the $n$ individuals would result in the correct outcome, with probability approaching one exponentially quickly as $n \rightarrow \infty$. Our interest in this article is in a variant of the process above where, after forming their initial opinions, the voters update their decisions based on some interaction with their neighbors in a social network. Our main example is "majority dynamics", in which each voter adopts the most popular opinion among its friends. The interaction repeats for some number of rounds and is then followed by a population-wide plurality vote. The question we tackle is that of "efficient aggregation of information": in which cases is the better alternative chosen with probability approaching one as $n \rightarrow \infty$ ? Conversely, for which sequences of growing graphs does aggregation fail, so that the wrong alternative gets chosen with probability bounded away from zero? We construct a family of examples in which interaction prevents efficient aggregation of information, and give a condition on the social network which ensures that aggregation occurs. For the case of majority dynamics we also investigate the question of unanimity in the limit. In particular, if the voters' social network is an expander graph, we show that if the initial population is sufficiently biased towards a particular alternative then that alternative will eventually become the unanimous preference of the entire population.
\end{abstract}

\footnotetext{
E. Mossel - J. Neeman

UC Berkeley, Berkeley, CA, USA

e-mail: mossel@stat.berkeley.edu

J. Neeman

e-mail: joeneeman@gmail.com

E. Mossel · O. Tamuz ( $\varangle)$

Weizmann Institute of Science, Rehovot, Israel

e-mail: omer.tamuz@weizmann.ac.il
} 
Keywords Social networks - Aggregation of information - Majority dynamics · Discrete Fourier analysis

\section{Introduction}

The mathematical study of voting systems began as early as 1785 , when the Marquis de Condorcet [5] observed what is essentially a special case of the weak law of large numbers: suppose there is a large population of voters, and each one independently votes "correctly" with probability $p>1 / 2$. Then as the population size grows, the probability that the outcome of a majority vote is "correct" converges to one. Thus, information is "efficiently aggregated".

In this work, we study a simple model of voter interaction, in which voters choose an independent random opinion initially, but then modify that opinion iteratively based on what their friends think. Thus correlation between votes is introduced "naturally," through interaction. Our main example of interaction is majority dynamics, where at each round each voter adopts the opinion of the majority of its neighbors. The basic question that we address is that of efficient information aggregation: for which modes of interaction is information aggregated efficiently, and for which is it not?

Additionally, we study some conditions for the achievement of unanimity when the graph of social ties is an expander and agents use majority dynamics.

\subsection{Model}

We consider an election in which a finite set $V$ of voters must choose between $q \geq 2$ alternatives, which we will take to be the elements of $[q]=\{0,1, \ldots, q-1\}$. The voters are connected by an undirected social network graph $G=(V, E)$. Denote the neighbors of $v \in V$ by $N_{v}$.

Each voter $v \in V$ will be initialized with a preference $X_{v}(0) \in[q]$, picked independently from a distribution $\mathbf{P}$ over $[q]$.

At time $t \in\{1, \ldots, T\}, v$ will update her opinion to $X_{v}(t)$ based on her friends' opinions at times $t-1$ and earlier. At time $T$, an election will take place and a winner $Y$ will be declared. Note that $Y$ is a deterministic function of the initial votes $\left(X_{v}(0)\right)_{v \in V}$.

A simple and important example is majority dynamics where $q=2$ : At each iteration of the dynamics, each individual $v$ sets her vote to equal the most popular vote among her neighbors in the previous iteration (we elaborate below on the handling of ties).

$$
X_{v}(t)=\operatorname{argmax}_{a \in\{0,1\}}\left|\left\{w \mid X_{w}(t-1)=a, w \in N_{v}\right\}\right| .
$$

At some large time $T$ an election by plurality takes place, so that the winner is

$$
Y=\operatorname{argmax}_{a \in\{0,1\}}\left|\left\{v \mid X_{v}(T)=a\right\}\right| .
$$

Note that the majority rule (or more generally the plurality rule, in the case of more than two alternatives) is fair and monotone: it is fair in the sense that is does not, as an election system, treat one alternative differently than another; it is invariant to a renaming of the alternatives. It is monotone in the sense that having extra supporters cannot hurt an alternative's case.

As a generalization of majority dynamics, we allow any updating of opinions and any election system, provided that they are fair and monotone. For example, an individual may give more weight to some of her friends than to others, and the final election could be an Electoral College system. In Sect. 6 we further relax the fairness condition. 


\subsection{Overview of the results}

\subsubsection{Social types}

Our study of information aggregation will utilize the idea of social type: we divide the voters $V$ into a partition $\mathcal{A}$ of social types, and ask that any two voters of the same social type play the same role in the election process. More precisely we require that if the labels are removed from all individuals then it is impossible to tell apart two individuals of the same social type. This shall be rigorously defined in Sect. 2 .

In the case of majority dynamics, social types are induced by the automorphisms ${ }^{1}$ of the graph $G: u, w \in V$ are of the same social type if there exists an automorphism $\tau$ of $G$ such that $\tau(u)=w$. Intuitively, this means that in an unlabeled drawing of $G$ it is impossible to say which is $u$ and which is $w ; u$ and $w$ are of the same social type if they play the same role in the geometry of $G$, and hence play the same role in the election process. A graph in which all vertices are of the same social type is called transitive.

\subsubsection{Aggregation of information}

Without loss of generality, we will assume that alternative 0 is the best alternative, and that the initial opinion of each voter is slightly biased towards alternative 0 : we take $X_{v}(0)$ to be a multinomial random variable such that $\mathbf{P}\left(X_{v}(0)=0\right)>\mathbf{P}\left(X_{v}(0)=j\right)$ for any $j \neq 0$.

Although this bias could be very small, the law of large numbers guarantees that with enough voters, the outcome of a plurality vote at time 0 would choose the correct alternative, except with exponentially low probability. We refer to this property as efficient aggregation of information.

In Sect. 4, we ask whether information is still efficiently aggregated if we hold the vote at time $T$ instead, after allowing the agents to interact. One of our main results (stated formally in Theorem 4.1 below for the general case of monotone dynamics and in Theorem 2.1 for the special case of majority dynamics) is that information is efficiently aggregated when each social type has many members. In particular, we show that the probability of choosing the correct alternative approaches one as the size of the smallest social type approaches infinity, with a polynomial dependence.

This implies that in majority dynamics on a transitive graph, in which case all voters are of the same social type, the outcome of the final vote will be zero, except with probability that decreases polynomially with the number of voters.

\subsubsection{Lack of aggregation}

Perhaps surprisingly, aggregation may fail when the condition requiring increasing size of each social type is not met. Indeed, in Sect. 4.1 we provide an example with $q=2$ alternatives, majority dynamics and a final majority vote, which results in the wrong outcome, with constant probability regardless of the size of the population.

An example given by Berger [4] can also be used for this purpose. We give our example since we feel that it is simpler and gives additional intuition as to why aggregation may fail.

$\overline{{ }^{1} \text { An automorphism of a graph } G}=(V, E)$ is a bijection $h: V \rightarrow V$ such that $(v, u) \in E \leftrightarrow(h(v), h(u)) \in E$ 


\subsubsection{Wider agreement, unanimity and expanders}

In Sect. 6, we ask when, following $T$ periods of interaction, a large part of the population is in agreement.

Focusing on the case $q=2$ and majority dynamics, we show that the proportion of the population that votes for alternative zero at time $T$ is at least as large as the initial bias towards alternative zero.

We push the agreement threshold to its extreme in Sect. 7, where we show that if the social network is an expander graph, the mode of interaction is based on plurality, and there is enough initial bias, then eventually the entire population will agree on alternative zero.

\subsection{Related work}

Our work is closely related to work of Kalai [16] who studies social choice using tools of discrete Fourier analysis. Kalai proves that any binary unbiased and monotone election system aggregates information efficiently, given that all the voters have low influence on the outcome.

Our work expands on this work in several directions: first, we elucidate the role of voters types in this setup by showing that having large number of voters of each type implies aggregation and that without this condition aggregation may not occur. Second, we go beyond the binary world and explore general outcome spaces. Finally the questions of higher thresholds and unanimity were not considered before.

Kanoria and Montanari [18] study majority dynamics with two alternatives on regular (infinite) tree graphs, giving conditions which lead to convergence to unanimity. Their work can also be interpreted as a study of a zero temperature spin glasses, a model also studied by Howard [13] on 3-regular trees and Fontes et al. [8] on $\mathbb{Z}^{d}$.

Berger [4] gives an example of a series of graphs in which majority dynamics results in the adoption, by all individuals, of the opinion of the individuals in a constant size group, provided they all agree. Thus these graphs could serve in place of our example (Sect. 4.1), showing how aggregation fails when there is a small social type. We provide our example for completeness, and because it is somewhat simpler.

Our work is related to the widely studied family of Gossip-based protocols on networks (see, e.g., Bawa et al. [3], Kempe et al. [19], and a survey by Shah [25]). The goal there is to design and/or analyze distributed, repeated algorithms for the aggregation of information on networks. For example, in the classical DeGroot model [6] agents "vote" with a real number, which they calculate at each iteration by averaging the votes of their neighbors from the previous iteration. The agents all converge to the same number, which is a good approximation of the average of the initial votes only if degrees are low [11], or if, indeed, the size of the smallest social type is large. This model is fairly easy to analyze, since the votes in each iteration are a linear function of the votes in the previous iteration. Majority dynamics is a natural discretization of this process, but has proven to be more resistant to analysis. Indeed the non-linearity of the dynamics results not only in major technical challenges but also in different behaviors of the two models.

Another related strain of models is that of Bayesian learning. Here the agents optimize their votes to those which are the most likely to be correct, given a prior over correct alternatives, an initial private signal and the votes of their neighbors in previous rounds (see, e.g., [2,22]). Perhaps surprisingly, this dynamic is not necessarily monotone and therefore its analysis requires different tools. The agents' calculations there are more complicated, and hence more difficult to analyze. On the other hand, the optimality of the agents' actions makes 
the model amenable to martingale arguments, which don't apply in the case of majority dynamics.

Our main proof uses tools from the field of Fourier analysis of Boolean functions on the discrete hypercube. In particular we use and extend results of Kahn, Kalai and Linial [14], Friedgut and Kalai [9], a strong version of the KKL theorem by Talagrand [26] and a recent generalization by Kalai and Mossel [17].

\section{Definitions and results}

We begin this section by defining the well known model of majority dynamics on social networks, and state our main result (Theorem 2.1) for this model. We then generalize the model to monotone dynamics, and restate our main result (Theorem 4.1) in this generality. Note that Theorem 2.1 is an immediate consequence of Theorem 4.1, since majority dynamics are a special case of monotone dynamics.

\subsection{Majority dynamics}

Let $V$ be a finite set of individuals. Let $G=(V, E)$, an undirected finite graph, represent the network of social connections of $V$. We denote the neighbors of $v \in V$ by $N_{v}$. We allow $G$ to contain self-loops, so that $v$ may or may not belong to $N_{v}$.

Let $X_{v}(t) \in\{0,1\}$ denote $v$ 's vote at time $t \in\{0, \ldots, T\}$. Let each $X_{v}(0)$ be chosen from some distribution $\mathbf{P}$ over $\{0,1\}$, independently and identically for all $v \in V$. Note that once the initial votes $\left(X_{v}(0)\right)_{v \in V}$ are chosen, the process is deterministic.

At times $t>0, v$ updates its vote to equal the majority opinion of its neighbors in the previous round. If the number of neighbors is even then we either add or remove $v$ itself to the set of neighbors $N_{v}$, to avoid ties.

$$
X_{v}(t)=\operatorname{argmax}_{a \in\{0,1\}}\left|\left\{w \mid X_{w}(t-1)=a, w \in N_{v}\right\}\right| .
$$

After some number of rounds $T$ an election by majority takes place. We denote the winner by $Y_{T}$ :

$$
Y_{T}=\operatorname{argmax}_{a \in\{0,1\}}\left|\left\{v \mid X_{v}(T)=a\right\}\right| .
$$

To avoid ties in the final election, we assume $|V|$ is odd.

We next define social types. Recall that $\tau: V \rightarrow V$ is a graph automorphism of $G=$ $(V, E)$ if $(u, v) \in E \leftrightarrow(\tau(u), \tau(v)) \in E$. We say that $u$ and $v$ are of the same social type if there exists a graph automorphism that maps $u$ to $v$. Informally, this means that $u$ and $v$ play the same role in the geometry of the graph; it is impossible to tell which is which if the labels are removed from the vertices. It is easy to see that "being of the same social type" is an equivalence relation. We denote by $\mathcal{A}(G)$ the partition of the vertices of $G$ into social types. We denote by $m(G)$ the size of the smallest social type:

$$
m(G)=\min _{A \in \mathcal{A}(G)}|A| .
$$

Our main result in this section is that information is aggregated efficiently, provided that each social type has many members. To state our result, we first define the efficiency of an aggregation procedure. Let $\mathbf{P}_{\delta}$ be the probability distribution on $\{0,1\}$ such that $\mathbf{P}_{\delta}(0)=$ $\frac{1}{2}(1+\delta)$ and $\mathbf{P}_{\delta}(1)=\frac{1}{2}(1-\delta)$. Then the efficiency $\mu_{\delta}(G, T)$ of majority dynamics on $G$ 
up to time $T$ is

$$
\mu_{\delta}(G, T)=\mathbf{P}_{\delta}\left[Y_{T}=0\right] .
$$

Note that in a slight abuse of notation we use $\mathbf{P}_{\delta}$ to denote both the distribution over $\{0,1\}$ from which $X_{v}(0)$ is chosen, and the measure on $\left(X_{v}(t)\right)_{v \in V, 1 \leq t \leq T}$ and $Y_{T}$ which is induced by $\mathbf{P}_{\delta}$.

Our main result for this section is the following:

Theorem 2.1 There exists a universal constant $C>0$ such that for any graph $G$

$$
\mu_{\delta}(G, T) \geq 1-C \exp \left(-C \frac{\delta \log m(G)}{\log (1 / \delta)}\right) .
$$

In particular, $\mu_{\delta}(G)$ approaches one as $m(G)$ tends to infinity. Note that the bound does not depend on $T$. This theorem is a special case of Theorem 4.1, which is stated below.

In the other direction, we provide an example showing what can go wrong when $m\left(G_{n}\right)$ does not grow to infinity.

Theorem 2.2 For any $\delta>0$, there exists a sequence of graphs $G_{n}$, whose sizes converge to infinity, such that

$$
\sup _{n} \sup _{T \geq 1} \mu_{\delta}\left(G_{n}, T\right)<1 .
$$

That is, there is some $\epsilon>0$ such that for any $n$ and $T$ the probability of choosing the wrong alternative is at least $\epsilon$.

\subsection{Monotone dynamics}

In this section we extend the definitions and results of the previous section to a large class of update rules and election systems, and a choice between more than two alternatives.

Let $[q]=\{0,1, \ldots, q-1\}$ be the set of alternatives. The initial votes $X_{v}(0)$ are, as above, chosen i.i.d. from some $\mathbf{P}$, which is now a distribution over $[q]$. As before, the process is deterministic once the initial votes are chosen.

Let the history of $v$ 's neighborhood before time $t$ be denoted by $H_{v}(t)=\left(X_{w}(s)\right)_{s<t, w \in N_{v}}$. Then $[q]^{[t] \times N_{v}}$ is the set of possible histories of the neighborhood of $v$ before time $t$.

For each $a \in[q]$ and $k \in \mathbb{N}$ we define a relation $\geq_{a}$ on $[q]^{k}$ as follows. Let $x, x^{\prime} \in[q]^{k}$. We write $x^{\prime} \geq_{a} x$ if, for all $i \in[k]$ it holds that

$$
x_{i}^{\prime} \neq x_{i} \rightarrow x_{i}^{\prime}=a .
$$

Alternatively, if a vector of votes $x$ is changed to $x^{\prime}$ such that $x^{\prime} \geq_{a} x$, then for each $i$ either $x_{i}$ is unchanged, or it is changed to $a$. Note that when $q=2$ then $x^{\prime} \geq_{1} x$ reduces to the usual $x^{\prime} \geq x$, i.e., $x_{i}^{\prime} \geq x_{i}$ for $i \in[k]$.

For $0<t \leq T$, let $X_{v}(t)$ be determined as follows. Let the mode of interaction be a collection of functions $m_{v, t}:[q]^{[t] \times N_{v}} \rightarrow[q]$, with

$$
X_{v}(t)=m_{v, t}\left(H_{v, t}\right) .
$$

These functions are generalization of the majority function used in majority dynamics. As such, we require that they meet the following conditions:

1. They are fair, or symmetric with respect to the alternatives: for all permutations $\sigma$ on $[q]$ and all histories $h \in[q]^{[t] \times N_{v}}, \sigma\left(m_{v, t}(h)\right)=m_{v, t}(\sigma(h))$, where $\sigma(h)$ is the result of applying $\sigma$ to each element of $h$. 
2. They are monotone: for every pair $h, h^{\prime} \in[q]^{[t] \times N_{v}}$, if $m_{v, t}(h)=a$ and $h^{\prime} \geq_{a} h$ then $m_{v, t}\left(h^{\prime}\right)=a$.

An example would be majority dynamics, i.e., the case where $q=2,\left|N_{v}\right|$ is odd for all $v$, and $m_{v, t}$ is equal to the most popular opinion among $X_{w}(t-1)$, where $w \in N_{v}$. A different simple example is the case that $X_{v}(t)$ is simply equal to $X_{v}(t-1)$, unless all of $v$ 's neighbors agree in time $t-1$ on some alternative $a$, in which case $X_{v}(t)=a$. That is, the agents do not change their opinions unless their friends unanimously agree on a different opinion.

Following $T$ rounds of interaction, we apply an election system function $g:[q]^{V} \rightarrow[q]$ to $\left(X_{1}(T), \ldots, X_{|V|}(T)\right)$, to determine the chosen alternative $Y$ :

$$
Y=g\left(X_{1}(T), \ldots, X_{|V|}(T)\right) .
$$

This is again a generalization of a majority vote, and as such we require that $g$ satisfy the same fairness and monotonicity properties:

1. It is fair, or symmetric with respect to the alternatives: for all permutations $\sigma$ on $[q], \sigma(g(a))=g(\sigma(a))$, where $\sigma(a)$ is the result of applying $\sigma$ to each element of $a, a_{v}$.

2. It is monotone: for every pair $x, x^{\prime} \in[q]^{V}$, if $g(x)=a$ and $x^{\prime} \geq_{a} x$ then $g\left(x^{\prime}\right)=a$.

Examples of such functions are the simple plurality function and various recursive plurality (i.e., electoral college-like) functions. Another important example is the dictator function, in which $g(a)=a_{v}$, for some fixed $v$.

The whole process of social interaction and elections can be viewed as a single function from the original signals $\left\{X_{v}(0) \mid v \in V\right\}$ to $[q]$. We denote this function by $f:[q]^{V} \rightarrow[q]$, and call it the aggregation function, so that

$$
Y=g\left(X_{1}(T), \ldots, X_{|V|}(T)\right)=f\left(X_{1}(0), \ldots, X_{|V|}(0)\right) .
$$

Note that for brevity's sake we sometimes write the above as $Y=f(X)$. It is easy to see that the aggregation function $f$ has the same properties we that require from the election system $g$ : it is monotone and fair.

Finally, the concept of social types is, in the case of monotone dynamics, defined by the symmetries of the aggregation function, rather than those of the graph. We first define $\mathcal{H}(f)$, the symmetry group of the aggregation function, as the group of permutations $\tau$ on $V$ that satisfy the following condition: for every $a \in[q]^{V}$ it holds that $f(\tau(a))=f(a)$, where $\tau(a)_{v}=a_{\tau(v)}$.

It is easy to verify that $\mathcal{H}(f)$ is indeed a group, with composition as the operation: for any $\tau, \sigma \in \mathcal{H}(f)$ it holds that $f(\tau(\sigma(a)))=f(\sigma(a))=f(a)$, and hence $\tau \sigma \in \mathcal{H}(f)$. Also, $f(a)=f\left(\tau\left(\tau^{-1}(a)\right)\right)=f\left(\tau^{-1}(a)\right)$, and so $\tau^{-1}$ is also in $\mathcal{H}(f)$.

The set of Social types is simply $V / \mathcal{H}(f)$, the set of orbits of $V$ under the action of $\mathcal{H}(f)$. I.e., $\mathcal{A}(f)$ is the unique partition of $V$ such that $v, w \in V$ are of the same social type iff $\exists \tau \in \mathcal{H}(f)$ such that $\tau(v)=w$.

The definition of $m(G)$ now naturally becomes the following. Given an aggregation function $f:[q]^{V} \rightarrow[q]$, denote by $m(f)$ the size of the smallest social type induced by $f:$

$$
m(f)=\min _{A \in \mathcal{A}(f)}|A| .
$$

Our main result of this section, which is a strict generalization of that of the previous, is again that information is aggregated efficiently provided that each social type has many members. In the case of monotone dynamics, our definition of the efficiency of aggregation is the following. Let $\mathcal{P}_{\delta}$ be the set of probability distributions $\mathbf{P}$ on $[q]$ under which $\mathbf{P}(0) \geq$ 
$\mathbf{P}(i)+\delta$ for all $i=1, \ldots, q-1$. Then the efficiency $\mu_{\delta}(f)$ of a function $f:[q]^{n} \rightarrow[q]$ is defined by

$$
\mu_{\delta}(f)=\inf _{\mathbf{P} \in \mathcal{P}_{\delta}} \mathbf{P}[f(X)=0] .
$$

Our main result for this section is the following:

Theorem 4.1. Let $f:[q]^{V} \rightarrow[q]$ be a monotone and fair aggregation function, and let $m=m(f)$ be the size of the smallest social type. Then

$$
\mu_{\delta}(f) \geq 1-C_{q} \exp \left(-C_{q} \frac{\delta \log m}{\log (1 / \delta)}\right),
$$

for some $C_{q}$ that depends only on $q$.

Theorem 4.1 is a statement about functions $f$ such that $m(f)$ is large. For $q=2$ and odd $n$ it is easy to find examples of such functions - the majority function, for example. However, not for every value of $q, n$ and $m \leq n$ there exists a fair and monotone aggregation function $f_{q, n}$ such that $m=m\left(f_{q, n}\right)$. In particular, it is not clear for which values of $q$ and $n$ there exists a fair and monotone aggregation function $f_{q, n}$ that is transitive, i.e., $m\left(f_{q, n}\right)=n$.

The challenge is to break ties in a way that preserves fairness and transitivity, and indeed it seems that no simple, immediate examples exist. We provide the following example of a fair, transitive and monotone function, for any $q \geq 2$ and $n$ prime and larger than $q$. See further discussion in N. AhmadiPourAnari, unpublished manuscript, 2011.

Proposition 2.3 For all $q \geq 2$ and $n$ prime and strictly larger than $q$, there exists a monotone, fair and transitive aggregation function $f:[q]^{n} \rightarrow[q]$.

\subsection{Unanimity results}

Besides the question of which candidate would win a majority vote, there are other interesting events we could study. As an extreme case, we consider the question of eventual unanimity, the event that everyone converges to the same opinion. Here we consider any number of alternatives $q$, but specialize to the case where the mode of interaction is given by simple plurality. It is easy to construct examples showing that our earlier assumptions on the structure of the network do not imply that the whole electorate will eventually agree. Indeed, there could be a small clique of voters who are well-connected to each other but poorly connected to the rest of the population. These voters could forever maintain an opinion contrary to that of their peers. One way to avoid this situation is to ask that the social network be an expander graph.

Let $M$ be the adjacency matrix of a $d$-regular graph $G$. We say that $G$ is a $\lambda$-expander graph if the second-largest absolute eigenvalue of $M$ is no larger than $\lambda$.

Although we will not require any knowledge of expander graphs here, we refer the uninitiated reader to [12] for a survey on the topic. For now, it is enough to know that "good" expanders have $\lambda=O(\sqrt{d})$.

Theorem 2.4 Let $G_{n}$ be a sequence of d-regular $\lambda$-expanders whose size converges to infinity. Suppose that $\frac{\lambda}{d} \leq \frac{3}{16}$ and

$$
\mathbf{P}(0) \geq \mathbf{P}(i)+\frac{c \sqrt{\log q}}{\sqrt{d}}
$$

for all $i \neq 0$. For $v$ a vertex in $G_{n}$ let $X_{v}(0)$ be drawn i.i.d. from $\mathbf{P}$, and let the mode of interaction be majority dynamics. Then with probability converging to 1 as $n \rightarrow \infty$, there exists a time $T$ such that $X_{v}(T)=0$ for all $v \in V$. 
The dependency on $d$ in Eq. (1) is possibly not tight. In particular, if $q=2$ and the girth of $G_{n}$ tends to infinity with $n$, then a result of Kanoria and Montanari [18] implies that we can replace $\sqrt{d}$ by $d^{\alpha}$ for any $\alpha>0$.

\subsection{Higher threshold results}

As an intermediate step between majority and unanimity, we next consider the event that one candidate achieves a substantial margin of victory. For $q=2$, consider the election system $g_{n, \alpha}(x)=\mathbb{1}\left(\sum_{i} x_{i} \geq(1-\alpha) n\right)$. When $\alpha=1 / 2$, this is just the simple majority function. It is monotone and fair, and so Theorem 4.1 applies. When $\alpha>1 / 2$, however, $g_{n, \alpha}$ is no longer symmetric in the alternatives. We prove that the final bias is as large as the expected initial bias.

Theorem 2.5 Let $f_{n, \alpha}$ be a monotone aggregation function with election system $g_{n, \alpha}$ on the graph $G_{n}$ after running $T$ rounds of interaction. If $m\left(f_{n, \alpha}\right) \rightarrow \infty$ and $\alpha<\frac{1}{2}+\frac{\delta}{2}$ then for any $T \in \mathbb{N}$,

$$
\lim _{n \rightarrow \infty} \mu_{\delta}\left(f_{n, \alpha}\right)=1 .
$$

We remark that the proof of Theorem 2.5 shows that $\mu_{\delta}\left(f_{n, \alpha}\right)$ converges to one polynomially fast in $m\left(f_{n, \alpha}\right)$.

We do not believe that the relationship between $\alpha$ and $\delta$ is the best possible. Note that for the complete graph on $n$ nodes, one can take $\alpha$ exponentially close to one for any $\delta$. It is natural to guess that the worst dependence on $n$ occurs in a ring. For this case we show that one can take $\alpha$ as large as $1-(1-\delta)^{2} / 2$ when $\delta$ is large, and $\frac{1}{2}+\frac{5}{6} \delta$ when $\delta$ is small. This example is interesting because it shows that a small initial bias in voters' opinions can result in a larger bias by the time the votes are cast. We do not know whether such a phenomenon exists on a larger class of graphs.

\section{Discussion and future directions}

In this article we consider the question of aggregation of information in a natural model of dynamics on a social network. Our main result is that information is well aggregated when there are no "special" agents; that is, when every agent is equivalent to many others. This is a rather strong condition of egalitarianism which is easily destroyed by a minor change to the network (or the election system, in the case of general monotone dynamics). Furthermore, networks that satisfy this condition are highly structured and unlikely to emerge organically. Hence, a natural question is whether a weaker condition suffices to ensure efficient aggregation of information.

All known majority dynamics counterexamples in which aggregation fails involve sequences of graphs with growing degrees. We therefore propose the following conjecture.

Conjecture 3.1 (Efficient aggregation of information on bounded degree graphs). Let $G_{n}$ be a sequence of graphs whose size converges to infinity and whose vertices all have degree at most $d$. Then for all $\delta>0$

$$
\liminf _{n} \mu_{\delta}\left(G_{n}, T\right)=1 .
$$

In the case of two alternatives, almost any reasonable election system is monotone and fair. However, as indicated by Sect. 5, the universe of functions that are fair and monotone is 
rather restricted when the number of alternatives is greater than two. It would be interesting to extend this work to, for example, the case that agents "vote" by declaring their order of preference on all the candidates (rather than just their top choice), and the voting system is Condorcet compatible, or alternatively satisfies Independence of Irrelevant Alternatives (see [23] for a complete characterization of the latter class).

\section{Aggregation of information}

In this section, we will prove the following theorem, using the definitions of Sect. 2.2.

Theorem 4.1 Let $f:[q]^{V} \rightarrow[q]$ be a monotone and fair aggregation function, and let $m=m(f)$ be the size of the smallest social type. Then

$$
\mu_{\delta}(f) \geq 1-C_{q} \exp \left(-C_{q} \frac{\delta \log m}{\log (1 / \delta)}\right),
$$

for some $C_{q}$ that depends only on $q$.

The proof of this theorem relies on a "sharp threshold" theorem of Kalai and Mossel [17] (which is itself an extension of Talagrand's theorem [26] to the case $q>2$ ). Sharp threshold theorems go back to Margulis [20] and Russo [24], Friedgut-Kalai [9] and Kalai [15] apply sharp threshold theorems in contexts similar to this one. In fact, the result of [15] gives a weaker version of Theorem 4.1 in which each social type must have at least $n / o(\log n)$ members.

A crucial ingredient for sharp threshold results is the notion of influence, which we will define for a function $f:[q]^{n} \rightarrow\{0,1\}$. Let $\mathbf{P}$ be a probability measure on $[q]$, and denote also by $\mathbf{P}$ the corresponding product distribution over $[q]^{n}$. The influence of voter $i$ on a function $f:[q]^{n} \rightarrow\{0,1\}$ is

$$
I_{\mathbf{P}}^{i}(f)=\mathbf{E}_{\mathbf{P}} \operatorname{Var}_{\mathbf{P}}\left(f\left(X_{1}, \ldots, X_{n}\right) \mid X_{1}, \ldots, X_{i-1}, X_{i+1}, \ldots, X_{n}\right),
$$

where $\operatorname{Var}_{\mathbf{P}}$ is the variance with respect to the product measure $\mathbf{P}$.

Kalai and Mossel [17] prove the following inequality:

Theorem 4.2 Suppose that $\mathbf{P}(a) \geq \alpha>0$ for every $a \in[q]$. If $\max _{i} I_{\mathbf{P}}^{i}(f) \leq \epsilon$ then

$$
\sum_{i=1}^{n} I_{\mathbf{P}}^{i}(f) \geq C \log n \frac{\log (1 / \epsilon)-\log (1 / 4)}{\log (1 / \alpha)} \operatorname{Var}_{\mathbf{P}}(f)
$$

for a universal constant $C$.

Theorem 4.2 is the main technical ingredient in the Proof of Theorem 4.1. The main contribution of Theorem 4.1 over the applications of Theorem 4.2 which appeared in [17] is that Theorem 4.1 applies to functions over multiple social types.

Before proving Theorem 4.1 we will require a simple definition and Lemma. Let $\mathbf{P}$ be a probability distribution on $[q]$ such that $\mathbf{P}(0)>0$. Define the following family of distributions $\mathbf{P}_{x}$ (indexed by $x \in[0,1]$ ) as follows:

$$
\mathbf{P}_{x}(a)= \begin{cases}x & a=0 \\ (1-x) \mathbf{P}(a \mid a \neq 0) & a>0 .\end{cases}
$$

Note that $\mathbf{P}_{\mathbf{P}(0)}=\mathbf{P}$. 
Lemma 4.3 Let $\mathbf{P}$ be a probability distribution on $[q]$ such that $\mathbf{P}(0)=\mathbf{P}(1)+\delta$ for some $\delta>0$. Let $y$ be such that $\mathbf{P}_{y}(0)=\mathbf{P}_{y}(1)$. Then

$$
\mathbf{P}(0)-y \geq \delta / 2 \text {. }
$$

Proof We can solve for $y$ to find that $y=(\mathbf{P}(0)-\delta) /(1-\delta)$. Hence

$$
\mathbf{P}(0)-y=(1-\mathbf{P}(0)) \frac{\delta}{1-\delta} \geq \delta / 2,
$$

Where the inequality follows from the fact that since $\mathbf{P}(0)=\mathbf{P}(1)+\delta \leq 1-\mathbf{P}(0)+\delta$, it holds that $1-\delta \leq 2-2 \mathbf{P}(0)$.

We prove Theorem 4.1 below by calculating the derivative of $\mathbf{P}_{x}(f=0)$ with respect to $x$ and then integrating between $x=y$ and $x=\mathbf{P}(0)$. We thus interpolate between $\mathbf{P}_{y}$, in which, by definition, the probability of zero and $a$ are equal, and $\mathbf{P}\left(=\mathbf{P}_{\mathbf{P}(0)}\right)$, in which the probability of zero is larger by $\delta$ than the probability of $a$. This idea goes back to the very first appearances of sharp threshold theorems [20,24].

For a function $g$ and a probability measure $\mathbf{P}$, we will write $\mathbf{P}(g)$ for the expectation of $g$ under $\mathbf{P}$.

Proof of Theorem 4.1 Since the conclusion of the theorem is only weakened when $\delta$ is reduced, we can assume without loss of generality that there is some $i>0$ such that the inequality $\mathbf{P}(0) \geq \mathbf{P}(i)+\delta$ is tight; moreover, we may assume that this $i$ is 1 , and so $\mathbf{P}(1)+\delta=\mathbf{P}(0)$. Choose $y \in[0, \mathbf{P}(0)]$ so that $\mathbf{P}_{y}(0)=\mathbf{P}_{y}(1)$.

Let $g:[q]^{V} \rightarrow[q]$ be defined by $g=1_{(f=0)}$; that is, $g(a)=1$ iff $f(a)=0$. Suppose (for now) that $\mathbf{P}(b) \geq \frac{\delta}{2 q}$ for all $b \in[q]$, and so $\mathbf{P}_{x}(b) \geq \frac{\delta}{2 q}$ for all $s \leq t \leq \mathbf{P}(0)$ and all $b \in[q]$. Since $f$ is fair and monotone, $\mathbf{P}_{y}(g) \geq 1 / q$. Indeed, the event $\{f=0\}$ is monotone with respect to the order $\leq_{0}$, and for any transposition $\tau$ on $[q]$ which swaps 0 with some element $i$ in $1, \ldots, q-1$, the measure $\mathbf{P}$ stochastically dominates $\mathbf{P} \circ \tau$ with respect to the ordering $\leq_{0}$. Hence, $\mathbf{P}(f=0) \geq(\mathbf{P} \circ \tau)(f=0)$ which, by the fairness of $f$, is equal to $\mathbf{P}(f=\tau(0))=\mathbf{P}(f=i)$. Since $\sum_{i=0}^{q-1} \mathbf{P}(f=i)=1$, it follows that $\mathbf{P}(g)=\mathbf{P}(f=0) \geq 1 / q$. Using monotonicity again, $\mathbf{P}_{x}(g) \geq 1 / q$ for all $x \geq y$.

By Theorem 4.2, if $\epsilon_{x}:=\max _{i \in[n]} I_{\mathbf{P}_{x}}^{i}(g)$ then

$$
\sum_{i=1}^{n} I_{\mathbf{P}_{x}}^{i}(g) \geq \frac{C \log \left(1 / \epsilon_{x}\right)}{\log (2 q / \delta)} \operatorname{Var}_{\mathbf{P}_{x}}(g) \geq \frac{C \log \left(1 / \epsilon_{x}\right)}{q \log (2 q / \delta)} \mathbf{P}_{x}(1-g)
$$

for all $x \in[y, \mathbf{P}(0)]$. Now, recall that for $A \in \mathcal{A}$, if $i, j \in A$ then they play the same role in $f$ and in particular have the same influence. Hence $\sum_{i=1} I_{\mathbf{P}_{x}}^{i}(g) \geq m \epsilon_{x}$, since $|A| \geq m$ for any $A \in \mathcal{A}$. In particular, if $\epsilon_{x} \geq(\log m) / m$ then $\sum_{i} I_{\mathbf{P}_{x}}^{i}(g) \geq \log m$; on the other hand, if $\epsilon_{x} \leq(\log m) / m$ then the display above implies that

$$
\sum_{i} I_{\mathbf{P}_{x}}^{i}(g) \geq C_{q} \frac{\log m}{\log (1 / \delta)} \mathbf{P}_{x}(1-g),
$$

for some $C_{q}$ that depends only on $q$. This last inequality (Eq. 4) holds, therefore, in either case.

On the other hand, Lemma 2.3 of [17] (a generalization of Russo's formula) gives

$$
\frac{\partial \mathbf{P}_{x}(g)}{\partial t} \geq \sum_{i} I_{\mathbf{P}_{x}}^{i}(g)
$$


and so

$$
\frac{\partial \mathbf{P}_{x}(g)}{\partial t} \geq C_{q} \frac{\log m}{\log (1 / \delta)} \mathbf{P}_{x}(1-g)
$$

for all $x \in[y, \mathbf{P}(0)]$. Integrating between $y$ and $x$, we have

$$
\mathbf{P}_{x}(g) \geq 1-(1-1 / q) \exp \left(-C_{q} \frac{\log m}{\log (1 / \delta)}(t-s)\right)
$$

and so we conclude by setting $x=\mathbf{P}(0)$ and invoking Eq. (3).

Now, if the hypothesis $\mathbf{P}(b) \geq \frac{\delta}{2 q}$ fails then we construct $\tilde{\mathbf{P}}$ by $\tilde{\mathbf{P}}(0)=\mathbf{P}(0)-\delta / 4$ and $\tilde{\mathbf{P}}(b)=\mathbf{P}(b)+\frac{\delta}{4(q-1)}$ for $b \neq 0$. Setting $\tilde{\delta}=\delta / 2$, we see that $\tilde{\mathbf{P}}$ satisfies the hypothesis of the theorem (with $\delta$ replaced by $\tilde{\delta}$ ) and it also satisfies $\tilde{\mathbf{P}}(b) \geq \frac{\tilde{\delta}}{2 q}$. The proof goes through, then, and we can absorb the extra factor of 4 into the constant $C_{q}$.

\subsection{Where aggregation fails}

Let $q=2$ and suppose that both the interaction mode and the election system are given by simple majority votes. In this scenario, we prove Theorem 2.2 by giving an example with two social types, one of which has a constant size as $n \rightarrow \infty$. Information will not aggregate asymptotically in this example, and the reason for the failure will be the presence of the constant-sized social type. Note that an example given by Berger [4] can be used to prove the same result. We give our example since it is simpler and provides additional intuition as to why aggregation fails.

Since $q=2$, it will be more convenient to set $p=\frac{1}{2}+\frac{\delta}{2}=\mathbf{P}(0)$ and to write our example in terms of $p$ instead of in terms of $\delta$. Let $G_{n}=(A \cup B, E)$, where $|A|=1 /(1-p)$ and $|B|=n(1 /(1-p)+1)$. Then in particular the number of vertices in $G_{n}$ is at least $n$. We assume here that $1 /(1-p)$ is an integer.

Let each $a \in A$ be connected to each $b \in B$, and let none of the vertices in $A$ be connected to each other. The vertices in $B$ are arranged in $n$ cliques, each of size $1 /(1-p)+1$, and there are no edges between the cliques. Each vertex in $B$ has a self-loop.

The degree of the vertices in $B$ is odd, since each has edges to $2 /(1-p)+1$ nodes. To make the degrees in $A$ odd add a vertex that is connected to all vertices in $A$. An isolated vertex can be added to make the total number of vertices odd.

Henceforth we condition on the event that $X_{v}(0)=1$ for all $v \in A$. Note that this happens with probability $(1-p)^{|A|}=(1-p)^{1 /(1-p)}$.

Let $C$ be one of the cliques of $B$. If at least one vertex $w$ in $C$ votes 1 initially (at time $t=0)$ then all the vertices in $C$ will vote 1 in the next round $(t=1)$; each will have at least $1 /(1-p)+1$ neighbors $(\{w\} \cup A)$ that vote 1 and at most $1 /(1-p)$ neighbors $(B \backslash\{w\})$ that vote 0 . The probability that at least one vertex in $C$ votes 1 initially is $1-p^{1 /(1-p)}$, which is greater than $1-1 / e$, or about 0.63 . Hence the number of cliques in which all vertices will vote 1 at time one will be distributed $\operatorname{Binom}\left(n, 1-p^{1 /(1-p)}\right)$, which dominates the distribution Binom $(n, 0.6)$.

By Hoeffding's inequality, the probability that a majority of the cliques (and hence a majority of the vertices) will vote 1 at time one is at least $1-\exp (-0.02 n)$. Once this happens, the vertices in $A$ will all vote 1 in all future iterations, and so will these cliques. Hence for all $T \geq 2$ a majority vote will result in one.

The event that a majority of the cliques have a voter that initially votes 1 is independent of the event that all vertices in $A$ initially vote 1 . Hence both events happen with probability 
at least $(1-p)^{1 /(1-p)}(1-\exp (-0.02))$. Since this quantity is positive and independent of $n$, it follows that information does not aggregate and Theorem 2.2 is proved.

Berger [4] constructs an example of a family of graphs with $n$ vertices. In each graph there exists a set of at most 18 vertices (which he calls a dynamic monopoly), such that if all agents in this set initially vote identically then, in majority dynamics with two alternatives, all the agents converge to the initial vote of the dynamic monopoly. In particular, this implies that in this example, with probability at least $(1-p)^{18}$, aggregation fails for any $n$. This is another example of how aggregation can fail when a particular social type has a small size (in this case at most 18).

\section{The existence of monotone, fair and transitive aggregation functions}

Proposition 5.1 For all $q \geq 2$ and $n$ prime and strictly larger than $q$, there exists a monotone, fair and transitive aggregation function $f:[q]^{n} \rightarrow[q]$.

Proof Let $q \geq 2$ and let $n>q$ be prime. Let $f:[q]^{n} \rightarrow[q]$ be defined as follows.

For $a=\left(a_{0}, \ldots, a_{n-1}\right) \in[q]^{n}$ let $Q(a)$ be the set of alternatives that received the most votes. If $Q(a)=\{b\}$ is a singleton then let $f(a)=b$. Otherwise $|Q(a)| \geq 2$. Let $M(a) \subset[n]$ be the set of voters that voted for one of the alternatives in $Q(a)$. Note that $|M(a)| \neq n$, since otherwise each alternative received the same number of votes and so $|Q(a)|$ divides $n$, which is impossible since $n$ is prime. Also, $M(a)$ is clearly not the empty set, and so $|M(a)|$ is an invertible element of the field $\mathbb{Z}_{n}$. Let

$$
k(a)=\frac{1}{|M(a)|} \sum_{i \in M(a)} i=\frac{1}{|M(a)|} \sum_{a_{i} \in Q(a)} i
$$

where addition and division are taken over the field $\mathbb{Z}_{n}$. Note that $k(a)$ is the "average" position of a voter that voted for one of the votes that received the most votes. Let

$$
\ell(a)=\min \{0 \leq i<n: k(a)+i \in M(a)\},
$$

where again the sum $k(a)+i$ is taken over $\mathbb{Z}_{n}$. Finally, define

$$
f(a)=a_{k(a)+\ell(a)} .
$$

By definition $f(a) \in Q(a)$, and so $f$ is the plurality function with some tie breaking rule, and is therefore monotone. Also, none of the alternative names appear in its definition, and it is therefore fair. It remains to show that it is transitive. We do this by showing that for each $0 \leq i_{1} \leq i_{2}<n$ there exists a permutation $\tau=\tau_{i_{1}, i_{2}}$ on $[n]$ such that $\tau\left(i_{1}\right)=i_{2}$ and $f(\tau(a))=f(a)$, where $\tau(a)=\left(a_{\tau(0)}, \ldots, a_{\tau(n-1)}\right)$.

Let $\tau_{i_{1}, i_{2}}(i)=\tau(i)=i-i_{1}+i_{2} \bmod n$. Note that $Q(\tau(a))=Q(a)$ and that $M(\tau(a))=$ $\tau^{-1}(M(a))$, so that $|M(\tau(a))|=|M(a)|$. Hence

$$
\begin{aligned}
k(\tau(a)) & =\frac{1}{|M(\tau(a))|} \sum_{i \in M(\tau(a))} i \\
& =\frac{1}{|M(a)|} \sum_{i \in \tau^{-1}(M(a))} i .
\end{aligned}
$$


By a change of variables we get that

$$
\begin{aligned}
k(\tau(a)) & =\frac{1}{|M(a)|} \sum_{i \in M(a)} \tau^{-1}(i) \\
& =k(a)+i_{1}-i_{2} \\
& =\tau^{-1}(k(a))
\end{aligned}
$$

Next,

$$
\begin{aligned}
\ell(\tau(a)) & =\min \{0 \leq i<n: k(\tau(a))+i \in M(\tau(a))\} \\
& =\min \left\{0 \leq i<n: k(a)+i_{1}-i_{2}+i \in M(a)+i_{1}-i_{2}\right\} \\
& =\ell(a),
\end{aligned}
$$

and finally, since $\tau(i+j)=\tau(i)+j$ :

$$
\begin{aligned}
f(\tau(a)) & =a_{\tau(k(\tau(a))+\ell(\tau(a)))} \\
& =a_{\tau(k(\tau(a)))+\ell(a)} \\
& =a_{k(a)+\ell(a)}=f(a) .
\end{aligned}
$$

\section{On higher thresholds of agreement}

In this section we again specialize to the case of $q=2$ alternatives, and consider the question of when it can be shown that, after a number of rounds of fair and monotone dynamics, a large proportion of the population will agree on the correct alternative.

Consider the election system $g_{n, \alpha}(x)=\mathbb{1}\left(\sum_{i} x_{i} \geq(1-\alpha) n\right)$. When $\alpha=1 / 2$, this is simply the majority function, and so our earlier results apply, and under the appropriate conditions $Y=g\left(X_{1}(T), \ldots, X_{|V|}(T)\right)$ will equal zero with high probability. What about when $\alpha>1 / 2$ ? In this case $Y$ will equal zero only if an $\alpha$ fraction of the population votes zero at time $T$. When does this happen with high probability?

Since $g_{n, \alpha}$ satisfies the same transitivity properties as $g_{n, 1 / 2}$, the proof of Theorem 4.1 mostly still applies. At least, the "sharp threshold" part of the claim is still true: there is some $p^{*} \in(0,1)$ such that $\mathbf{P}(0)>p^{*}$ implies that $\mathbf{P}(Y=0) \rightarrow_{m\left(f_{n}\right)}$ 1. Since $g_{n, \alpha}$ is no longer anti-symmetric, however, we no longer know that the threshold occurs at $p^{*}=1 / 2$.

In this section, we will show that $p^{*} \leq \alpha$, but we will also give a simple example for which $p^{*}=1-O\left((1-\alpha)^{2}\right)$ as $\alpha \rightarrow 1$. Thus, there may be a large gap between our bound and the true behavior of $p^{*}$.

The first step is to obtain a lower bound on $\mathbf{E} \sum_{i} X_{v}(t)$. The argument here appeared in a course taught by the first author in Fall 2010, although it may have been known before then. In any case, we give a proof for completeness. For the rest of this section, $\mathbf{P}_{p}$ denotes the probability distribution on $\{0,1\}$ satisfying $\mathbf{P}_{p}(0)=p$, in which case $\delta=2 p-1$. As above, we also denote by $\mathbf{P}_{p}$ the distribution over $n$ i.i.d. random variables distributed $\mathbf{P}_{p}$.

Lemma 6.1 Let $f:\{0,1\}^{n} \rightarrow\{0,1\}$ be a monotone function with $\mathbf{P}_{1 / 2}(f=0) \geq \frac{1}{2}$. Then $\mathbf{P}_{p}(f=0) \geq p$ for all $p \in\left[\frac{1}{2}, 1\right]$. 
Note that equality holds for the function $f(x)=x_{i}$. In other words, every monotone function aggregates information at least as well as a dictator function. It is easy to construct less pathological examples that come arbitrarily close to achieving this bound.

Proof By the chain rule,

$$
\begin{aligned}
\frac{\partial \mathbf{P}_{p}(f)}{\partial p}= & \sum_{i=1}^{n} \mathbf{P}_{p}\left(f\left(X_{1}, \ldots, X_{i-1}, 0, X_{i+1}, \ldots, X_{n}\right)\right. \\
& \left.-f\left(X_{1}, \ldots, X_{i-1}, 1, X_{i+1}, \ldots, X_{n}\right)\right) \\
= & -\frac{1}{p(1-p)} \sum_{i=1}^{n} I_{P_{p}}^{i}(f) .
\end{aligned}
$$

By the Efron-Stein inequality [7], $\sum I^{i}(f) \geq \operatorname{Var}(f)$, with equality only if $f$ depends just on one coordinate. If $f$ depends just on one coordinate, then the proof is trivial, so we can suppose the contrary. Thus $\frac{\partial}{\partial p} \mathbf{P}_{p}(f)<-\frac{1}{p(1-p)} \operatorname{Var}_{\mathbf{P}_{p}}(f)$.

Suppose, for a contradiction, that $1-\mathbf{P}_{p}(f)=\mathbf{P}_{p}(f=0)<p$ for some $p>\frac{1}{2}$. Let $r$ be the infinum over all $p$ satisfying the previous sentence. Since $\mathbf{P}_{p}(f)$ is a smooth function of $p$, it follows that $\mathbf{P}_{r}(f)=1-r$ and so $\operatorname{Var}_{\mathbf{P}_{r}}(f)=r(1-r)$. Thus, $\left.\frac{\partial}{\partial p} \mathbf{P}_{p}(f)\right|_{p=r}<-1$, contradicting the assumption that $\mathbf{P}_{p}(f)>1-p$ for arbitrarily close $p>r$.

Note that for any vertex $v$ and any $t$, the conditions of the lemma hold for $f=X_{v}(t)$. Summing over all $v$, we obtain the following:

Corollary 6.2 Suppose that $X_{v}(0)$ are independent Bernoulli variables with mean $p \geq \frac{1}{2}$. Then, for any $t$,

$$
\mathbf{E} \sum_{v \in V} X_{v}(t) \leq(1-p)|V|
$$

Combining this with the proof of Theorem 4.1, we arrive at the promised bound on the location of the sharp threshold. By taking $m \rightarrow \infty$, Corollary 6.3 immediately implies Theorem 2.5.

Corollary 6.3 Let $f_{n}:[q]^{n} \rightarrow q$ be a sequence of aggregation functions with monotone and fair modes of interaction and election system $g_{n, \alpha}$ as defined above. Suppose that $\lim _{n \rightarrow \infty} m\left(f_{n}\right)=\infty$, and that $p>\alpha$. Then $\mathbf{P}_{p}(Y=0) \geq 1-m^{-C \epsilon^{2}}$ for some universal constant $C$.

Proof For the sake of brevity, denote $g_{n, \epsilon}=g_{n, \epsilon}\left(X_{1}(T), \ldots, X_{|V|}(T)\right.$ ) (which equals $Y$ for $\epsilon=\alpha)$. From the proof of Theorem 4.1, we have

$$
\frac{\partial \mathbf{P}_{p}\left(g_{n, \alpha}=0\right)}{\partial p} \geq C(\log m) \operatorname{Var}_{\mathbf{P}_{p}}\left(g_{n, \alpha}\right) .
$$

On the other hand, Corollary 6.2 gives us that for any $\epsilon>0$

$$
\mathbf{P}_{p}\left(g_{n, p-\epsilon}=0\right)=\mathbf{P}_{p}\left(\sum_{v \in V} X_{v}(t) \leq(1-p+\epsilon)|V|\right) \geq \epsilon
$$

and so $\operatorname{Var}_{\mathbf{P}_{p}}\left(g_{n, p-\epsilon}\right) \geq \epsilon \mathbf{P}_{p}\left(g_{n, p-\epsilon}\right)$ for any $\epsilon$.

Fix $\alpha<p$ and set $\epsilon=(p-\alpha) / 2$. Then for any $r \in[\alpha+\epsilon, p], \operatorname{Var}_{\mathbf{P}_{r}}\left(g_{n, \alpha}\right) \geq \epsilon \mathbf{P}_{r}\left(g_{n, \alpha}\right)$ and so we can solve the differential inequality 


$$
\frac{\partial \mathbf{P}_{r}\left(g_{n, \alpha}=0\right)}{\partial r} \geq C \epsilon(\log m) \mathbf{P}_{r}\left(g_{n, \alpha}\right)
$$

in the range $[p-\epsilon, p]$, with initial condition $\mathbf{P}_{p-\epsilon}\left(g_{n, \alpha}=0\right) \geq \epsilon$. We obtain

$$
\left.\mathbf{P}_{p}(Y=0)=\mathbf{P}_{p}\left(g_{n, \alpha}=0\right) \geq 1-(1-\epsilon) \exp \left(-C \epsilon^{2} \log m\right)\right) \geq 1-\exp \left(-C \epsilon^{2} \log m\right) .
$$

\subsection{An example: cycles}

Let $G_{n}$ be a cycle on $n$ vertices, where each vertex has a self-loop, and recall that $p=$ $\frac{1+\delta}{2}$. When the mode of interaction is majority dynamics, we can explicitly calculate the distribution of $\lim _{t \rightarrow \infty} X_{v}(t)$. We study voter aggregation on cycles for two reasons. Firstly, we obtain more precise answers than the general bound (Theorem 2.5) on higher thresholds, thereby demonstrating that Theorem 2.5 may not be sharp; specifically, we find that for cycles, $\mu_{\delta}\left(f_{n, \alpha}\right) \rightarrow 1$ for a larger range of $\alpha$ than those guaranteed to work by Theorem 2.5. Secondly, cycles are a natural example to study because they are in some sense the most poorly-connected transitive graphs. It is therefore natural to conjecture that cycles are the worst-performing graphs for higher thresholds, suggesting that perhaps Theorem 2.5 could be improved in general.

Of particular interest are the cases when $\delta \rightarrow 0$ or $\delta \rightarrow 1$; for small $\delta, \alpha<\frac{1}{2}+\frac{5}{6} \delta-\Omega\left(\delta^{3}\right)$ turns out to imply $\lim _{n \rightarrow \infty} \mu_{\delta}\left(f_{n, \alpha}\right) \rightarrow 1$, while for large $\delta$, if we set $\epsilon=1-\delta$, then $\alpha<1-\frac{1}{2} \epsilon^{2}$ is sufficient. Therefore, the bound in Theorem 2.5 is not tight: for $\delta$ close to zero, one can take $\alpha \approx \frac{1}{2}+\frac{5}{6} \delta$ while Theorem 2.5 only guarantees that $\alpha=\frac{1}{2}+\frac{1}{2} \delta$ will work; for $\delta$ close to $1, \alpha \approx 1-\frac{1}{2} \epsilon^{2}$ is sufficient, but Theorem 2.5 only allows $\alpha=1-\frac{1}{2} \epsilon$.

The analysis of the cycle is relatively simple because the eventual state of the voters can be easily foretold from the initial state. First of all, whenever two (or more) adjacent voters share the same opinion, they will retain that opinion forever. Moreover, strings of voters whose opinions alternate will gradually turn into strings of voters with the same opinion, as in the following example:

$$
\begin{array}{ccccccccccc}
\operatorname{time} t & \cdots & 1 & 1 & 0 & 1 & 0 & 1 & 0 & 0 & \cdots \\
\text { time } t+1 & \cdots & 1 & 1 & 1 & 0 & 1 & 0 & 0 & 0 & \cdots \\
\text { time } t+2 & \cdots & 1 & 1 & 1 & 1 & 0 & 0 & 0 & 0 & \cdots
\end{array}
$$

In fact, one can tell the eventual opinion of a voter $v$ with the following simple rule: let $V \geq 0$ be the smallest number such that $X_{v-V}=X_{v-V-1}$ and let $W \geq 0$ be the smallest number such that $X_{v+W}=X_{v+W+1}$ (assuming that such $V$ and $W$ exist, which will only fail to happen in the unlikely event that the whole cycle consists of alternating opinions). If $V \leq W$ then $X_{v}(t)=X_{v-V}(0)$ for all $t \geq V$. On the other hand, if $W \leq V$ then $X_{v}(t)=X_{v+W}(0)$ for all $t \geq W$. (If $V=W$ then $X_{v-V}(0)=X_{v+W}(0)$ because $X_{v-V}(0)=X_{v}(0)$ if and only if $V$ is even, and similarly for $W$.)

Proposition 6.4 Let $G$ be the cycle on $n$ vertices. Then for any $v$,

$$
\lim _{T \rightarrow \infty} \lim _{n \rightarrow \infty} \mathbf{P}\left(X_{v}(t)=0 \text { for all } t \geq T\right)=\frac{2 p^{2}-p^{3}}{1-p+p^{2}}=\frac{1}{2}+\frac{5 \delta-\delta^{3}}{6+2 \delta^{2}}=1-\frac{4 \epsilon^{2}-\epsilon^{3}}{8-4 \epsilon+2 \epsilon^{2}} .
$$

As we observed following Corollary 6.2, this implies that if $\alpha<\frac{1}{2}+\frac{5 \delta-\delta^{3}}{6+2 \delta^{2}}$ and the number of interaction rounds is sufficiently large (depending on $\alpha$ and $p$ ), then $\mu_{\delta}\left(f_{n, \alpha}\right) \rightarrow 1$. 
Proof For brevity, we will write $X_{v}$ instead of $X_{v}(0)$ for the initial state of vertex $v$. Instead of majority dynamics on the cycle, consider majority dynamics on $\mathbb{Z}$; we will see later that these are essentially the same when $n$ is large. We may assume without loss of generality that $v=0$. As in the discussion above, let $V \geq 0$ be minimal such that $X_{-V}=X_{-V-1}$ and let $W \geq 0$ be minimal such that $X_{W}=X_{W+1}$.

Let us first condition on $X_{0}(0)=0$. Consider the i.i.d. sequence

$$
Y_{k}=\left(X_{-2 k}, X_{1-2 k}, X_{2 k-1}, X_{2 k}\right) \in\{0,1\}^{4} .
$$

If $Y_{1}, \ldots, Y_{j}=(0,1,1,0)$ then the sequence $X_{-2 j}, \ldots, X_{2 j}$ consists of alternating zeros and ones, and so $V, W \geq 2 j$. Define $A_{0}, A_{1} \subset\{0,1\}^{4}$ by

$$
\begin{aligned}
& A_{0}=\{(a, b, c, d): b=0 \text { or } c=0\} \\
& A_{1}=\{(a, b, c, d): a=b=c=1 \text { or } b=c=d=1\}
\end{aligned}
$$

Note that $A_{0} \cap A_{1}=\emptyset$ and $\{0,1\}^{4} \backslash\left(A_{0} \cup A_{1}\right)=\{(0,1,1,0)\}$. Therefore, if $J$ is minimal such that $Y_{J} \neq(0,1,1,0)$ then $Y_{J}$ is in either $A_{0}$ or $A_{1}$. If $Y_{J} \in A_{0}$ then either $W=2 J-2$ and $X_{W}=0$ :

$$
\begin{array}{ccccccccc}
X_{0} & X_{1} & X_{2} & X_{3} & X_{4} & \cdots & X_{2 J-3} & X_{2 J-2} & X_{2 J-1} \\
0 & 1 & 0 & 1 & 0 & \cdots & 1 & 0 & 0
\end{array}
$$

or $V=2 J-2$ and $X_{-V}=0$ :

$$
\begin{array}{cccccccccc}
X_{-(2 J-1)} & X_{-(2 J-2)} & X_{-(2 J-3)} & \cdots & X_{-4} & X_{-3} & X_{-2} & X_{-1} & X_{0} \\
0 & 0 & 1 & \cdots & 0 & 1 & 0 & 1 & 0
\end{array}
$$

In either of these cases, $X_{0}(t)=0$ for all $t \geq 2 J-2$. Conversely, if $Y_{J} \in A_{1}$ then either $X_{W}=1$ or $X_{V}=1$ and $X_{0}(t)=1$ for all $t \geq 2 J-1$. Thus, (using the fact that $J$ and $Y_{J}$ are independent)

$$
\mathbf{P}\left(X_{0}(t)=0 \text { for all } t \geq T \mid X_{0}=0\right)=\mathbf{P}\left(Y_{J} \in A_{0}\right) \mathbf{P}(2 J-2 \leq T) .
$$

Since the $Y_{j}$ are i.i.d,

$$
\begin{aligned}
\mathbf{P}\left(Y_{J} \in A_{0}\right) & =\frac{\mathbf{P}\left(Y_{1} \in A_{0}\right)}{\mathbf{P}\left(Y_{1} \in A_{0} \cup A_{1}\right)} \\
& =\frac{2 p-p^{2}}{2 p-p^{2}+2(1-p)^{3}-(1-p)^{4}} \\
& =\frac{2 p-p^{2}}{1-p^{2}+2 p^{3}-p^{4}},
\end{aligned}
$$

where we have computed $\mathbf{P}\left(Y_{1} \in A_{i}\right)$ by the inclusion/exclusion formulas

$$
\begin{aligned}
\mathbf{P}\left(Y_{1} \in A_{0}\right)= & \mathbf{P}\left(X_{-1}=0\right)+\mathbf{P}\left(X_{1}=0\right)-\mathbf{P}\left(X_{-1}=X_{1}=0\right) \\
\mathbf{P}\left(Y_{1} \in A_{1}\right)= & \mathbf{P}\left(X_{-2}=X_{-1}=X_{1}=1\right)+\mathbf{P}\left(X_{-1}=X_{1}=X_{2}=1\right) \\
& -\mathbf{P}\left(X_{-2}=\cdots=X_{2}=1\right) .
\end{aligned}
$$

The case for $X_{0}=1$ is similar: we define

$$
\begin{aligned}
& A_{0}^{\prime}=\{(a, b, c, d): a=b=c=0 \text { or } b=c=d=0\} \\
& A_{1}^{\prime}=\{(a, b, c, d): b=1 \text { or } c=1\} .
\end{aligned}
$$


If $J^{\prime}$ is minimal such that $Y_{J^{\prime}} \neq(1,0,0,1)$ then $Y_{J^{\prime}} \in A_{0}^{\prime}$ implies $X_{0}(t)=0$ for $t \geq 2 J^{\prime}-1$, while $Y_{J^{\prime}} \in A_{1}^{\prime}$ implies $X_{0}(t) \rightarrow 1$ for $t \geq 2 J^{\prime}-2$. Since $\mathbf{P}\left(Y_{1} \in A_{0}^{\prime}\right)=2 p^{3}-p^{4}$ and $\mathbf{P}\left(Y_{1} \in A_{1}^{\prime}\right)=2(1-p)-(1-p)^{2}$, we have

$$
\frac{\mathbf{P}\left(X_{0}(t)=0 \text { for all } t \geq T \mid X_{0}=1\right)}{\mathbf{P}(2 J-1 \leq T)}=\frac{\mathbf{P}\left(Y_{1} \in A_{0}^{\prime}\right)}{\mathbf{P}\left(Y_{1} \in A_{0}^{\prime} \cap A_{1}^{\prime}\right)}=\frac{2 p^{3}-p^{4}}{1-p^{2}+2 p^{3}-p^{4}} .
$$

To transition back from dynamics on $\mathbb{Z}$ to dynamics on the $n$-cycle, note that the event $\left\{X_{0}(t)=0\right.$ for all $\left.t \geq T\right\}$ is the same event on $\mathbb{Z}$ and on the $n$-cycle, provided that $n>2 T$. In particular, (5) and (6) imply that

$$
\lim _{n \rightarrow \infty} \mathbf{P}\left(X_{0}(t)=0 \text { for all } t \geq T \mid X_{0}=0\right)=\mathbf{P}(2 J-2 \leq T) \frac{2 p-p^{2}}{1-p^{2}+2 p^{3}-p^{4}}
$$

for majority dynamics on the $n$-cycle (and similarly conditioned on $X_{0}=1$, using (7). Since $\lim _{T \rightarrow \infty} \mathbf{P}(2 J-2 \leq T)=1$,

$$
\lim _{T \rightarrow \infty} \lim _{n \rightarrow \infty} \mathbf{P}\left(X_{0}(t)=0 \text { for all } t \geq T \mid X_{0}=0\right)=\frac{2 p-p^{2}}{1-p^{2}+2 p^{3}-p^{4}} .
$$

(and similarly conditioned on $X_{0}=1$ ). Finally,

$$
\begin{aligned}
\mathbf{P}\left(X_{0}(t)=0 \text { for all } t \geq T\right) & =p \mathbf{P}\left(X_{0}(t)=0 \text { for all } t \geq T \mid X_{0}=0\right)+(1-p) \mathbf{P}\left(X_{0}(t)\right. \\
& \left.=0 \text { for all } t \geq T \mid X_{0}=1\right) \rightarrow \frac{2 p^{2}-p^{3}}{1-p+p^{2}}
\end{aligned}
$$

as $T, n \rightarrow \infty$. The formulas in terms of $\delta$ and $\epsilon$ are obtained by substituting $p=\frac{1+\delta}{2}=1-\frac{\epsilon}{2}$.

\section{Expander graphs converge to unanimity}

7.1 Majority dynamics with two alternatives

In this section we again consider the case that $q=2$ and majority dynamics (i.e., each voter adopts the majority opinion of its neighbors), with a population wide majority vote at time $T$. To avoid the issue of ties, we assume that $\left|N_{v}\right|$ is odd for all $v$ and that $n$ is odd.

Let $G$ be a graph and $M$ its adjacency matrix, so that $M_{v u}$ is 1 if $(u, v) \in E$ and zero otherwise. We say that $G$ is a $\lambda$-expander if the second-largest absolute eigenvalue of $M$ is at most $\lambda$ (cf. [12]). Expander graphs have particularly nice properties under the iterated majority dynamics. One reason for this is that in an expander graph, the number of edges between disjoint sets $A$ and $B$ of vertices is almost completely determined by the cardinalities of $A$ and $B$. We state this formally in Lemma 7.1 below.

Denote $E(A, B)=1_{A}^{T} M 1_{B}$, where $A$ and $B$ be sets of vertices. Note that if $A$ and $B$ are disjoint then $E(A, B)$ is the number of edges between $A$ and $B$, and if $A$ and $B$ are not disjoint, then $E(A, B)$ double-counts edges from $A \cap B$ to itself. Alternatively, $E(A, B)$ is the number of "edge-ends" of edges with one end in $A$ and another in $B$.

Recall that a graph is $d$-regular if all vertices have degree $d$, i.e., $\left|N_{v}\right|=d$ for all $v \in V$.

Lemma 7.1 (Expandermixing lemma (cf. [1])) If $G$ is a d-regular $\lambda$-expander with $n$ vertices then

$$
\left|E(A, B)-\frac{|A||B| d}{n}\right| \leq \lambda \sqrt{|A||B|}
$$

for every $A, B \subset G$. 
It follows easily from the expander mixing lemma that medium-sized majorities are unstable under iterated majority dynamics. That is, if a reasonable majority of people prefer one outcome then very quickly a large majority of people will prefer that outcome.

Proposition 7.2 Let $q=2$, let $n$ be odd, let $G$ be a $d$-regular $\lambda$-expander with $d$ odd, and let the mode of interaction be majority dynamics with a majority vote at time $T$.

Let $N_{0}(t)$ be the number of agents that vote 0 at time $t$ and let $N_{1}(t)$ be the number of agents that vote 1 . If $N_{0}(t) \geq N_{1}(t)+\alpha$ then $N_{1}(t+1) \leq \frac{2 \lambda^{2}}{\alpha^{2} d^{2}} n$.

Proof Let $A_{0}(t)$ be the set of agents that vote 0 at time $t$, and define $A_{1}(t)$ similarly. Then, by the nature of majority dynamics, every $v \in A_{1}(t+1)$ has more than half of its neighbors in $A_{1}(t)$. Summing over every $v \in A_{1}(t+1)$, we have $E\left(A_{1}(t+1), A_{1}(t)\right) \geq E\left(A_{1}(t+\right.$ $\left.1), A_{0}(t)\right)$. By applying the expander mixing lemma to both sides,

$$
\frac{N_{1}(t+1) N_{0}(t) d}{n}-\lambda \sqrt{N_{1}(t+1) N_{0}(t)} \leq \frac{N_{1}(t+1) N_{1}(t) d}{n}+\lambda \sqrt{N_{1}(t+1) N_{1}(t)} .
$$

Rearranging, and since $N_{0}(t)-N_{1}(t) \geq \alpha n$,

$$
\alpha \sqrt{N_{1}(t+1)} \leq \frac{\lambda}{d}\left(\sqrt{N_{1}(t)}+\sqrt{N_{0}(t)}\right) \leq \frac{\lambda}{d} \sqrt{2 n} .
$$

Applying the proposition twice, we see that an imbalance of $\frac{4 \lambda n}{d}$ implies that a large, stable majority will form within one time-step.

Corollary 7.3 If $N_{0}(t) \geq N_{1}(t)+\frac{4 \lambda n}{d}$ and $\frac{\lambda}{d} \leq \frac{3}{16}$ then $N_{1}(s) \leq \frac{n}{8}$ for all $s \geq t+1$.

Proof Taking $\alpha=\frac{4 \lambda}{d}$ in Proposition 7.2, we have $N_{1}(t+1) \leq \frac{n}{8}$. Then $N_{0}(t+1) \geq$ $N_{1}(t+1)+\frac{3 n}{4} \geq \frac{4 \lambda n}{d}$ and so we can continue applying Proposition 7.2 indefinitely with $\alpha=\frac{4 \lambda}{d}$.

In order to show that a complete consensus is eventually achieved, we will use a result of [10], who proved that majority dynamics will eventually enter a cycle with period at most two.

Proposition 7.4 If $\frac{\lambda}{d} \leq \frac{3}{16}$ and $N_{0}(t)-N_{1}(t) \geq \frac{4 \lambda n}{d}$ for some $t$, then majority dynamics converge to all zero.

Proof Since majority dynamics converge to a cycle with period at most two, we can divide the vertices of $G$ into four sets: $A_{00}$ is the set of nodes that converge to $0, A_{11}$ is the set that converge to one, with $A_{01}$ and $A_{10}$ being the two sets of nodes that eventually alternate between zero and one. By Corollary 7.3, $\left|A_{11}\right|+\max \left\{\left|A_{01}\right|,\left|A_{10}\right|\right\} \leq \frac{n}{8}$, and so $\left|A_{00}^{c}\right|=$ $\left|A_{11}\right|+\left|A_{01}\right|+\left|A_{10}\right| \leq \frac{n}{4}$. By the expander mixing lemma,

$$
\left|E\left(A_{00}^{c}, A_{00}^{c}\right)\right| \leq\left|A_{00}^{c}\right|^{2} \frac{d}{n}+\lambda\left|A_{00}^{c}\right| \leq\left|A_{00}^{c}\right|\left(\frac{d}{4}+\lambda\right) .
$$

On the other hand, $\left|E\left(A_{00}, A_{00}^{c}\right)\right|+\left|E\left(A_{00}^{c}, A_{00}^{c}\right)\right|=d\left|A_{00}^{c}\right|$ and so $\left|E\left(A_{00}, A_{00}^{c}\right)\right| \geq$ $\left|A_{00}^{c}\right|\left(\frac{3 d}{4}-\lambda\right)$. Since $\lambda \leq d / 4,\left|E\left(A_{00}, A_{00}^{c}\right)\right| \geq \frac{d}{2}\left|A_{00}^{c}\right|$. Supposing that $A_{00}^{c}$ is non-empty, there must be at least one vertex $v \in A_{00}^{c}$ with more than half of its neighbors in $A_{00}$. But then the definition of majority dynamics would imply that $v$ converges to zero, a contradiction. Thus $A_{00}^{c}$ must be empty, and all agents converge to zero. 
In particular, a random $d$-regular graph has $\lambda=O(\sqrt{d})$ with high probability. Therefore, if we start with an initial bias such that $\mathbf{P}(0)-\frac{1}{2} \gtrsim d^{-1 / 2}$ then iterated majority on a random $d$-regular graph will converge to all zero with high probability.

\subsection{Plurality dynamics on expanders}

The results of the previous section can be extended with little effort to the case of more than two alternatives. The main obstacle in making this extension is specifying the resolution of ties. With two alternatives, we avoid the possibility of ties in majority dynamics simply by requiring each vertex to have odd degree. With more than two alternatives, the simplest way to avoid ties is to perturb the edge weights slightly so that they are rationally independent. ${ }^{2}$ Our expansion assumptions can be easily extended to the weighted case: let $M$ be the weighted adjacency matrix of $G$ and assume that all of its entries on or above the main diagonal are rationally independent of one another. Let $d$ be the largest absolute eigenvalue of $M$ and let $\lambda$ be the second-largest. Note that if $M$ was constructed by perturbing the edge weights of a random regular graph, then $d$ will be approximately the degree of the graph and $\lambda$ will be $O(\sqrt{d})$.

With the assumptions above, Lemma 7.1 holds exactly as it was stated above, and so the proof of Proposition 7.2 applies also.

Proposition 7.5 For $a \in[q]$, let $N_{a}(t)$ be the number of people that vote a at time $t$. If $N_{a}(t) \geq \frac{1+\alpha}{2} n$ then $N_{a}(t+1) \geq n\left(1-\frac{2 \lambda^{2}}{\alpha^{2} d^{2}}\right)$

To get an extension of Proposition 7.4, we first need to extend the periodicity result [10] to the case of several alternatives. This extension uses exactly the same argument as [10], but we include it for completeness.

Proposition 7.6 On a weighted graph with no ties, iterated plurality dynamics converge to a cycle of length at most two.

Proof Consider the quantity

$$
J_{v}(t)=\sum_{a \in[q]}\left(\left(1_{\left\{X_{v}(t+1)=a\right\}}-1_{\left\{X_{v}(t-1)=a\right\}}\right) \sum_{w \sim v} e_{w v} 1_{\left\{X_{w}(t)=a\right\}}\right),
$$

where $e_{w v}$ is the weight of the edge between $v$ and $w$. Note that $J_{v}(t) \geq 0$ with equality if, and only if, $X_{v}(t+1)=X_{v}(t-1)$. Indeed, if $X_{v}(t+1)=X_{v}(t-1)$ then $J_{v}(t)=0$ trivially, so suppose that $X_{v}(t+1)=a$ and $X_{v}(t-1)=b \neq a$. Then

$$
J_{v}(t)=\sum_{\left\{w \sim v: X_{w}(t)=a\right\}} e_{w v}-\sum_{\left\{w \sim v: X_{w}(t)=b\right\}} e_{w v} .
$$

Since $X_{v}(t+1)=a$ and the edge weights are chosen to ensure that ties never happen, this implies that $J_{v}(t)>0$.

Now consider $J(t)=\sum_{v} J_{v}(t)$. Note that if we define

$$
L(t)=\sum_{v} \sum_{w \sim v} \sum_{a \in[q]} e_{w v} 1_{\left\{X_{v}(t+1)=a\right\}} 1_{\left\{X_{w}(t)=a\right\}}
$$

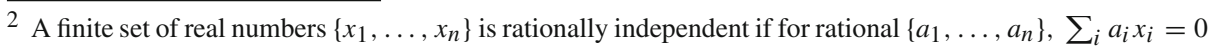
implies that the $a_{i}$ 's are all equal to zero. 
then $J(t)=L(t)-L(t-1)$. Since the state space of the dynamics is finite and the dynamics are deterministic, the process eventually (by time $T$, say) converges to a cycle (of period $k$, say). Then

$$
\sum_{t=T+1}^{T+k} J(t)=\sum_{t=T+1}^{T+k} L(t)-\sum_{t=T}^{T+k-1} L(t)=0,
$$

since the states are identical at time $T$ and $T+k$, and thus $L(T)=L(T+k)$. Since $J(t) \geq 0$ for every $t$, it follows that $J(T+1)=0$. Then $J_{v}(T+1)=0$ for every $v$ and so the state at time $T+2$ is identical to the state at time $T$.

With Proposition 7.6 in hand, the rest of the proof of Proposition 7.4 goes through in the $q$-alternative case. We only note that we need to replace $A_{01}$ by the set $A_{a *}=\left\{v: X_{v}(2 t)=\right.$ $a \neq X_{v}(2 t+1)$ for large enough $\left.t\right\}$.

Proposition 7.7 If $\frac{\lambda}{d} \leq \frac{3}{16}$ and $N_{a}(t) \geq n\left(\frac{1}{2}+\frac{2 \lambda}{d}\right)$ for some $t$ then the plurality dynamics converge to $a$.

In particular, if we take a random $d$-regular graph and perturb each edge weight by at most $n^{-3}$, then the second eigenvalue will hardly change, so we will still have $\lambda=O(\sqrt{d})$. If $\mathbf{P}\left(X_{v}(0)=a\right) \geq \mathbf{P}\left(X_{v}(0)=b\right)+\frac{c \sqrt{\log q}}{\sqrt{d}}$ for every $b \neq a$ then at time $t=1$, with high probability most of the vertices will prefer $a$ and Proposition 7.7 will imply that the plurality dynamics will converge to all $a$.

\subsection{A stronger result for expanders with large girth}

In Sect. 7.1 we proved that in majority dynamics with two alternatives, an initial bias of $d^{-1 / 2}$ is sufficient (on a random $d$-regular graph) for consensus in the limit. Kanoria and Montanari [18] showed that on an infinite $d$-regular tree, the required bias is much smaller as a function of $d$ :

Theorem 7.8 (Kanoria and Montanari) Let $v$ be a vertex in an infinite d-regular tree. For any $\beta>0$ and all sufficiently large $d$, if $\mathbf{P}(0) \geq \frac{1}{2}+d^{-\beta}$ then with probability one, $X_{v}(t)=0$ for all sufficiently large $t$.

Using this, it is easy to improve our earlier bias requirement for consensus from $\mathbf{P}(0)-\frac{1}{2} \gtrsim$ $d^{-1 / 2}$ to $\mathbf{P}(0)-\frac{1}{2} \gtrsim d^{-\beta}$ for any $\beta>0$ :

Corollary 7.9 For every $d$, let $G_{n, d}$ be a sequence of $d$-regular $\lambda$-expanders with $\frac{\lambda}{d} \leq \frac{3}{16}$, such that the girth of $G_{n, d}$ tends to infinity with $n$. For any $\beta>0$, if $p \geq \frac{1}{2}+d^{-\beta}$ then for all sufficiently large $d$, with high probability $($ as $n \rightarrow \infty)$ the iterated majority process on $G_{n, d}$ will converge to all zero.

Proof Choose $d$ large enough (depending on $\beta$ ) so that Theorem 7.8 applies, then choose $T$ large enough so that $\mathbf{P}\left(X_{v}(T)=0\right) \geq \frac{1}{2}+\frac{C}{\sqrt{d}}$ on the $d$-regular tree, for some constant $C$ to be determined. By choosing $n$ large enough, we can ensure that the girth of $G_{n, d}$ is larger than $T$; thus $\mathbf{P}\left(X_{v}(T)=0\right) \geq \frac{1}{2}+\frac{C}{\sqrt{d}}$ for every $v \in G_{n, d}$. Then the expected fraction of nodes that are 0 by time $T$ is at least $\frac{1}{2}+\frac{C}{\sqrt{d}}$, since at time $T$ each node only depends on the initial values of nodes within a ball of radius $T$. Since the number of such nodes is bounded as $n \rightarrow \infty$, McDiarmid's inequality [21] implies that with high probability, at least $\frac{1}{2}+\frac{C-1}{\sqrt{d}}$ fraction of nodes are zero at time $T$. If we choose $C$ large enough, Proposition 7.4 implies that the dynamics converge to all zero. 
Acknowledgements We would like to thank Miklos Racz for his careful reading of the manuscript and his suggestions. Elchanan Mossel is supported by a Sloan fellowship in Mathematics, by BSF Grant 2004105, by NSF Career Award (DMS 054829), by ONR Award N00014-07-1-0506 and by ISF Grant 1300/08. Omer Tamuz is supported by ISF Grant 1300/08. Omer Tamuz is a recipient of the Google Europe Fellowship in Social Computing, and this research is supported in part by this Google Fellowship.

\section{References}

1. Alon, N., \& Spencer, J. (2008). The probabilistic method (Vol. 73). New York: Wiley-Interscience.

2. Bala, V., \& Goyal, S. (1998). Learning from neighbours. Review of Economic Studies, 65(3), 595-621.

3. Bawa, M., Garcia-Molina, H., Gionis, A., \& Motwani R. (2003). Estimating aggregates on a peer-to-peer network. submitted for publication.

4. Berger, E. (2001). Dynamic monopolies of constant size. Journal of Combinatorial Theory, Series B, 83(2), 191-200.

5. Condorcet, J.-A.-N. (1785). Essai sur l'application de l'analyse à la probabilité des décisions rendues à la pluralité des voix. De l'Imprimerie Royale.

6. DeGroot, M. H. (1974). Reaching a consensus. Journal of the American Statistical Association, 69(345), $118-121$.

7. Efron, B., \& Stein, C. (1981). The jackknife estimate of variance. The Annals of Statistics, 9(3), 586-596.

8. Fontes, L., Schonmann, R., \& Sidoravicius, V. (2002). Stretched exponential fixation in stochastic ising models at zero temperature. Communications in Mathematical Physics, 228(3), 495-518.

9. Friedgut, E., \& Kalai, G. (1996). Every monotone graph property has a sharp threshold. Proceedings of the American Mathematical Society, 124(10), 2993-3002.

10. Goles, E., \& Olivos, J. (1980). Periodic behaviour of generalized threshold functions. Discrete Mathematics, 30(2), 187-189.

11. Golub, B., \& Jackson, M. (2010). Naive learning in social networks and the wisdom of crowds. American Economic Journal: Microeconomics, 2(1), 112-149.

12. Hoory, S., Linial, N., \& Wigderson, A. (2006). Expander graphs and their applications. Bulletin of the American Mathematical Society, 43(4), 439-561.

13. Howard, C. (2000). Zero-temperature ising spin dynamics on the homogeneous tree of degree three. Journal of Applied Probability, 37, 736-747.

14. Kahn, J., Kalai, G., \& Linial, N. (1988). The influence of variables on boolean functions. In Proceedings of the 29th Annual Symposium on Foundations of Computer Science (pp. 68-80).

15. Kalai, G. (2001). Social choice and threshold phenomena. Discussion Paper Series.

16. Kalai, G. (2004). Social indeterminacy. Econometrica, 72, 1565-1581.

17. Kalai, G., \& Mossel, E. (2010). Sharp thresholds for non-boolean functions and social choice theory. Preprint.

18. Kanoria, Y., \& Montanari, A. (2009). Majority dynamics on trees and the dynamic cavity method. Arxiv, preprint arXiv:0907.0449.

19. Kempe, D., Dobra, A., \& Gehrke, J. (2003). Gossip-based computation of aggregate information. In Proceedings of the 44th Annual Symposium on Foundations of Computer Science (pp. 482-491). New York: IEEE.

20. Margulis, G. (1977). Probabilistic characteristic of graphs with large connectivity. Problems of Information Transmission, 10, 174-179.

21. McDiarmid, C. (1989). On the method of bounded differences. Surveys in Combinatorics, 141(1), 148188.

22. Mossel, E., Sly, A., \& Tamuz, O. (2012). Asymptotic learning on Bayesian social networks. Preprint at http://arxiv.org/abs/1207.5893

23. Mossel, E., \& Tamuz, O. (2012). Complete characterization of functions satisfying the conditions of arrows theorem. Social Choice and Welfare, 39(1), 127-140.

24. Russo, L. (1982). An approximate zero-one law. Probability Theory and Related Fields, 61(1), 129-139.

25. Shah, D. (2009). Gossip algorithms. Foundations and Trends in Networking, 3(1), 1-125.

26. Talagrand, M. (1994). On Russo's approximate zero-one law. The Annals of Probability, 22(3), 15761587. 